(2) Open Access Full Text Article

\title{
Impact of Comorbidities and Commonly Used Drugs on Mortality in COPD - Real-World Data from a Primary Care Setting
}

This article was published in the following Dove Press journal: International Journal of Chronic Obstructive Pulmonary Disease

\author{
Jens Ellingsen (iD) \\ Gunnar Johansson (iD) ${ }^{2}$ \\ Kjell Larsson ${ }^{3}$ \\ Karin Lisspers ${ }^{2}$ \\ Andrei Malinovschi (iD) ${ }^{4}$ \\ Björn Ställberg $\mathbb{D}^{2}$ \\ Marcus Thuresson (iD) \\ Christer Janson $\mathbb{D}^{1}$ \\ 'Department of Medical Sciences, \\ Respiratory, Allergy and Sleep Research, \\ Uppsala University, Uppsala, Sweden; \\ ${ }^{2}$ Department of Public Health and Caring \\ Sciences, Family Medicine and Preventive \\ Medicine, Uppsala University, Uppsala, \\ Sweden; ${ }^{3}$ Integrative Toxicology, National \\ Institute of Environmental Medicine, \\ Karolinska Institutet, Stockholm, Sweden; \\ ${ }^{4}$ Department of Medical Sciences, Clinical \\ Physiology, Uppsala University, Uppsala, \\ Sweden; ${ }^{5}$ Statisticon AB, Uppsala, \\ Sweden
}

Correspondence: Jens Ellingsen Department of Medical Sciences, Respiratory, Allergy and Sleep Research, Akademiska Sjukhuset, Uppsala SE-75 I 85 , Sweden

Tel +46 I8 6III I393

$\mathrm{Fax}+46186110228$

Email jens.ellingsen@medsci.uu.se
Background: Life expectancy is significantly shorter for patients with chronic obstructive pulmonary disease (COPD) than the general population. Concurrent diseases are known to infer an increased mortality risk in those with COPD, but the effects of pharmacological treatments on survival are less established. This study aimed to examine any associations between commonly used drugs, comorbidities and mortality in Swedish real-world primary care COPD patients.

Methods: Patients with physician-diagnosed COPD from a large primary care population were observed retrospectively, utilizing primary care records and mandatory Swedish national registers. The time to all-cause death was assessed in a stepwise multiple Cox proportional hazards regression model including demography, socioeconomic factors, exacerbations, comorbidities and medication.

Results: During the observation period (1999-2009) 5776 (32.5\%) of 17,745 included COPD patients died. Heart failure (hazard ratio [HR]: 1.88, 95\% confidence interval [CI]: 1.74-2.04), stroke (HR: 1.52, 95\% CI: 1.40-1.64) and myocardial infarction (HR: 1.40, 95\% CI: 1.24-1.58) were associated with an increased risk of death. Use of inhaled corticosteroids (ICS; HR: 0.79, 95\% CI: 0.66-0.94), beta-blockers (HR: 0.86, 95\% CI: 0.76-0.97) and acetylsalicylic acid (ASA; HR: 0.87, 95\% CI: 0.77-0.98) was dose-dependently associated with a decreased risk of death, whereas use of long-acting muscarinic antagonists (LAMA; HR: $1.33,95 \%$ CI: 1.14-1.55) and N-acetylcysteine (NAC; HR: 1.26, 95\% CI: 1.08-1.48) were dose-dependently associated with an increased risk of death in COPD patients.

Conclusion: This large, retrospective, observational study of Swedish real-world primary care COPD patients indicates that coexisting heart failure, stroke and myocardial infarction were the strongest predictors of death, underscoring the importance of timely recognition and treatment of comorbidities. A decreased risk of death associated with the use of ICS, betablockers and ASA, and an increased risk associated with the use of LAMA and NAC, was also found.

Keywords: observational, LAMA, inhaled corticosteroids, beta-blockers, acetylsalicylic acid, chronic obstructive pulmonary disease

\section{Plain Language Summary}

Patients with chronic obstructive lung disease (COPD) die earlier than healthy people. Having certain other diseases (for instance heart disorders) at the same time as COPD is common and known to increase the risk of death. It is not certain whether medication can improve survival or not. There is a need for deeper knowledge of the connection between other diseases, drugs and COPD deaths. 
Our research team analyzed data from medical records and registers on almost 18,000 COPD patients from Sweden. We found that heart diseases and stroke were connected to an increased risk of death. We also found that a certain bronchodilator drug type (long-acting muscarinic antagonists, LAMA) was connected to an increased risk. The following drugs were connected to a decreased risk of COPD death:

- inhaled corticosteroids (common anti-inflammatory drugs used for COPD and asthma),

- beta-blockers (used for various heart diseases and high blood pressure),

- aspirin (used to prevent heart disease).

This study showed that it is important for physicians treating COPD patients to also be attentive for other diseases. There is still a need for further studies investigating the roles of different drugs to prevent COPD deaths.

\section{Introduction}

Chronic obstructive pulmonary disease (COPD) is one of the world's major causes of death with an estimated 2.9 million deaths annually. ${ }^{1}$ In Sweden, overall life expectancy for patients with COPD has been shown to be 8.3 years shorter than that of the general population. ${ }^{2}$ Comorbidities influence morbidity and mortality in COPD, ${ }^{3}$ as exemplified by the increased mortality risk associated with cardiovascular disease (CVD), lung cancer, diabetes and depression or anxiety, among others. ${ }^{4-7}$ The more comorbidities a COPD patient has, the higher is the risk of early death.

Whether pharmacological treatment can impact COPD mortality is debated. There is conflicting evidence regarding the effect of inhaled corticosteroids (ICS) on COPD mortality when assessed in randomized controlled trials (RCTs). ${ }^{8,9}$ Systematic reviews also found no significant difference in mortality between long-acting beta-2-agonists (LABA) and placebo, ${ }^{10}$ or between long-acting muscarinic antagonists (LAMA) and placebo. ${ }^{11}$

Treatment of comorbid diseases might influence COPDrelated mortality. Retrospective, observational data associate beta-blockers ${ }^{12}$ and angiotensin-converting enzyme inhibitors (ACEi) or angiotensin-II receptor blockers (ARB) ${ }^{13}$ with a decreased COPD mortality; however, no RCTs examining these relationships exist. A meta-analysis reported decreased mortality for COPD patients on antiplatelet therapy. ${ }^{14}$ An RCT evaluating statins in COPD patients with no other indications for statin treatment did not show any significant effect on mortality as compared to placebo. ${ }^{15}$
Currently, most of the knowledge of factors influencing COPD mortality has been acquired from RCTs with a highly select study-population or studies made in secondary care settings, ${ }^{4-6,16}$ indicating populations with more severe COPD. There are only limited data from realworld primary care COPD cohorts on the effect of comorbidities on mortality, ${ }^{7,17}$ and to our knowledge no such studies examining the effect of drug-use on mortality. The aim of this observational, retrospective study was to increase our knowledge on the influence of comorbidities and pharmacological treatment on COPD mortality, by combining data from electronic patient records with data from Swedish national registers on a large real-world cohort of primary care COPD patients.

\section{Methods}

This was an observational, retrospective, population-based study, in which several previous analyses have been made. ${ }^{2,18-21}$ Primary care records were linked by the Swedish National Board of Health and Welfare to data from mandatory Swedish national registers. The linked and anonymized database was held and managed by the Department of Public Health and Caring Sciences, Uppsala University, Uppsala, Sweden. The study was registered at www.clinical trials.gov (clinical trial identifier NCT01146392).

\section{Data Sources}

A total of 76 primary healthcare centers were included in the study, covering approximately 800,000 individuals (about $8 \%$ of the Swedish population). To get a sample representative for the entire population, healthcare centers from both urban and rural areas, of various sizes and run by both private and public providers were included.

Medical records data from the primary healthcare centers included were extracted with an established software system (Pygargus Customized eXtraction Program, CXP; Pygargus AB, Stockholm, Sweden). ${ }^{22}$ Data extracted were date of birth, gender and diagnoses by the Swedish adaptation of ICD-10 (International Classification of Diseases, 10th revision) codes. From the mandatory Swedish National Patient Register data were collected on inpatient care (admission and discharge dates, main and secondary diagnoses) and outpatient secondary care (number of contacts, diagnoses). Moreover, data were extracted from the Cause of Death Register (date and cause[s] of death) and from the Swedish Prescribed Drugs Register (drug prescription data from both hospital and primary care: prescription date, collection date, drug type, dose, pack size). Extraction of data from the 
registers was made by the Swedish National Board of Health and Welfare. Information on marital status, educational level and yearly income were obtained from the Swedish governmental authority Statistics Sweden (SCB, Stockholm, Sweden).

\section{Study Population}

The cohort included all patients with COPD (J44, according to ICD-10) diagnosed by a physician. In order to reduce the risk of immortal time bias we only included patients diagnosed during the study period, 1st January 1999 to 31 st December 2009. Patients with a COPD-diagnosis in the Cause of Death Register only were excluded, but there were no other exclusion criteria. The index date was defined as the date of first COPDdiagnosis, and patients were followed until 31st December 2009, emigration or death.

\section{Study Measures Mortality}

The main outcome of interest was all-cause mortality during the study period. Date of death was collected from the Cause of Death Register.

\section{Socioeconomic Factors}

Included in this study were baseline data on age, yearly income, marital status (defined as either single or cohabiting) and educational level, defined as low or high $(<10$ or $\geq 10$ years of total education).

\section{Exacerbations}

Exacerbations were defined by one or more of the following events: COPD-related hospitalizations (ICD-10 code J44 as main diagnosis or $\mathrm{J} 44.0 / \mathrm{J} 44.1$ as secondary diagnosis), emergency visits (ICD-10 code J44.0/J44.1 in outpatient hospital care) or collection of prescribed oral steroids (Anatomical Therapeutic Chemical Classification System [ATC] code H02AB) or antibiotics (ATC codes J01AA and J01CA, encompassing antibiotics recommended for and commonly used against COPD exacerbations). ${ }^{18}$ To avoid including the same exacerbation more than once in the analysis, exacerbation events as defined above occurring within 14 days were defined as a single exacerbation. Included in the analyses were exacerbations occurring from two years before baseline until the end of follow-up. Since an ICD-10 code of COPD hospitalization or exacerbation would have constituted inclusion in the study, only drug prescription events were regarded prior to baseline.

\section{Comorbidities}

Concurrent diseases were identified by ICD-10 codes: E10-15, diabetes; F32-33, depression; I10-15, hypertension; I21, acute myocardial infarction (MI); I20-25, ischemic heart disease (IHD); I50, heart failure (HF); I61-64, stroke; M80-82, osteoporosis; and S02, S12, S22, S32, S42, S52, S62, S72, S82, S92, fractures. Asthma was defined as the existence of the diagnosis (J45 according to ICD-10) prior to index date (ie, date of COPD diagnosis), and pneumonia was defined as the occurrence of ICD-10 codes $\mathrm{J} 10-18$ during the two-year period preceding index date. The Charlson comorbidity index, ${ }^{23}$ adapted for ICD10 by Quan et al, ${ }^{24}$ of 17 diseases including COPD was used to calculate a score based upon the existence and severity of the diseases at the time of or within the twoyear period preceding COPD diagnosis. More severe or a higher number of diseases yield a higher Charlson comorbidity index score. ${ }^{23,24}$

\section{Drug Usage}

Drug usage from two years before index date until the end of follow-up was analyzed and updated on a yearly basis. To examine any exposure-dependency, drug usage was assessed both as any use of and as relative exposure to the drug class of interest. Relative exposure was calculated based on prescribed dose, prescription frequency, pack size and collected items, generating a cumulative number of defined daily doses which was then divided by the total number of days of exposure to the drug during the preceding three years. The following drug classes were included (ATC code[s]): ICS (R03BA); LABA (R03AC12 and R03AC13); fixed combination of ICS and LABA (R03AK); LAMA (R03BB, in all patients tiotropium bromide since it was the only LAMA available in Sweden during the study period); N-acetylcysteine (NAC; R05CB); oral steroids (H02AB); bisphosphonates (M05BA); statins (C10AA); acetylsalicylic acid (ASA; B01AC06); angiotensinconverting enzyme inhibitors (ACEi; C09A and C09B), beta-blockers (C07); angiotensin-II receptor blockers (ARB; C09C and C09D); diuretics (C03); and selective serotonin reuptake inhibitors (SSRI; N06AB).

\section{Other Variables}

Data on smoking status and lung function were only available from a small part of the population $(12.3 \%$ and $12.8 \%$, respectively) and was therefore not included in the analyses. No data was available on vaccination status or blood eosinophil count. 


\section{Statistical Analyses}

Descriptive analyses were done comparing COPD patients that deceased during the study period with those who were alive at the end of follow-up. Cox proportional hazards regression models were used to analyze time to death, producing hazard ratios (HR) presented with a $95 \%$ confidence interval (CI). Firstly, all potential risk factors were analyzed separately (simple Cox regression). Secondly, all factors with a p-value below 0.2 in the simple Cox regression model were entered into a stepwise multiple Cox proportional hazards regression model. The model with the lowest Akaike information criterion value was chosen as the final model, in which not all risk factors entered into the analysis were included. Multicollinearity was not formally assessed, since the stepwise multiple Cox proportional hazards regression model usually excludes one of two strongly correlated variables.

The following variables were included in the primary analyses. Baseline characteristics at time of COPD diagnosis: gender, age, marital status, income, and educational level. Also included as baseline characteristics were Charlson comorbidity index score and a diagnosis of pneumonia or asthma prior to COPD diagnosis. In addition, a number of time-dependent factors were added to the analyses: drug usage - relative exposure (detailed above), drug usage - any use, exacerbations, hypertension, HF, MI, IHD, stroke, diabetes, osteoporosis, fractures and depression. Exacerbations and fractures were analyzed on a per-event basis. Patients were censored when they left the study due to death or emigration. Latest time point alive was used to censor patients who were alive at the end of follow-up.

A sensitivity analysis was done where the association between use of LAMA and mortality was analyzed after stratifying the LAMA-treated patients for concomitant use of ICS (yes or no). Cox regression was used in these analyses and the estimates were adjusted for age, gender and Charlson comorbidity index score.

Data management and statistical analyses were performed using SAS version 9.3 (SAS Institute Inc, Cary, NC, US) and $\mathrm{R}$ version 3.5.0 (The R Foundation, Vienna, Austria).

\section{Results}

\section{Cohort Characteristics}

From a cohort of 17,745 patients with a diagnosis of COPD followed for a total of 64,306 person-years, $5776(36.7 \%)$ died during the study period. Those who died were older at the time of diagnosis, more often male and more often single, and they had a lower level of education and a lower yearly income than those who were alive at end of follow-up (Table 1). Exacerbations during the two-year period preceding baseline were less common among those who died during the study period, as were hypertension. All other comorbidities studied, however, were more common at baseline among those who died, indicating a group of patients with a higher total disease-

Table I Baseline Characteristics of the Cohort at Time of COPD Diagnosis, Comparing Patients Alive at End of FollowUp to Those Who Deceased During the Study Period (n (\%) Unless Otherwise Stated)

\begin{tabular}{|c|c|c|c|}
\hline & \multirow{2}{*}{$\begin{array}{l}\text { Alive } \\
(\mathrm{N}=11,969)\end{array}$} & \multirow{2}{*}{$\begin{array}{l}\text { Deceased } \\
(N=5776)\end{array}$} & \multirow[t]{2}{*}{ p-value } \\
\hline & & & \\
\hline Male gender & $5346(44.7)$ & $3026(52.4)$ & $<0.001$ \\
\hline Age (years, mean $\pm S D$ ) & $64.5 \pm 10.9$ & $74.6 \pm 9.5$ & $<0.001$ \\
\hline Higher education ${ }^{\mathrm{a}}$ & $6209(54.6)$ & $|26|(25.8)$ & $<0.001$ \\
\hline Marital status: single $\mathrm{e}^{\mathrm{a}}$ & $3219(28.3)$ & $1863(38.1)$ & $<0.001$ \\
\hline Yearly income $^{a}$ (SEK, median) & 131,000 & 111,000 & $<0.001$ \\
\hline Born in Sweden & $\begin{array}{l}10,162 \\
(85.1)\end{array}$ & $5155(88.6)$ & $<0.001$ \\
\hline Exacerbation $(s)^{\mathrm{b}}$ & $4435(37.1)$ & $1673(29.0)$ & $<0.001$ \\
\hline Pneumonia(s) ${ }^{\mathrm{b}}$ & $1024(8.6)$ & $696(12.1)$ & $<0.001$ \\
\hline Heart failure & $689(5.8)$ & $1081(18.7)$ & $<0.001$ \\
\hline Myocardial infarction & $478(4.0)$ & $418(7.2)$ & $<0.001$ \\
\hline Ischemic heart disease & $864(7.2)$ & $732(12.7)$ & $<0.001$ \\
\hline Stroke & $624(5.2)$ & $561(9.7)$ & $<0.001$ \\
\hline Hypertension & $3117(26.0)$ & $1406(24.3)$ & 0.015 \\
\hline Diabetes & $1027(8.6)$ & $647(11.2)$ & $<0.001$ \\
\hline Osteoporosis & $295(2.5)$ & $228(4.0)$ & $<0.001$ \\
\hline Depression & $1352(11.3)$ & $419(7.3)$ & $<0.001$ \\
\hline Asthma & $2346(19.6)$ & $1038(18.0)$ & 0.010 \\
\hline $\begin{array}{l}\text { Charlson Comorbidity Index score } \\
\text { (mean; median [interquartile } \\
\text { range]) }\end{array}$ & $0.86 ; 0[0,1]$ & $\begin{array}{l}1.33 ; 1 \\
{[0,2]}\end{array}$ & $<0.001$ \\
\hline ICS & $2922(24.4)$ & $1340(23.2)$ & 0.078 \\
\hline LAMA & $2549(21.3)$ & $1465(25.4)$ & $<0.001$ \\
\hline LABA & $137 \mid(11.5)$ & $649(11.2)$ & 0.687 \\
\hline ICS/LABA, fixed combination & $1993(16.7)$ & $606(10.5)$ & $<0.001$ \\
\hline $\mathrm{N}$-acetylcysteine & $5358(44.8)$ & 2304 (39.9) & $<0.001$ \\
\hline Oral steroids & $2525(21.1)$ & $1060(18.4)$ & $<0.001$ \\
\hline Acetylsalicylic acid & $2310(19.3)$ & $1330(23.0)$ & $<0.001$ \\
\hline $\mathrm{ACE \textrm {i }}$ & $1530(12.8)$ & $847(14.7)$ & 0.001 \\
\hline Beta-blockers & $2848(23.8)$ & $1242(21.5)$ & 0.001 \\
\hline ARB & $1230(10.3)$ & $424(7.3)$ & $<0.001$ \\
\hline Diuretics & $2653(22.2)$ & $1906(33.0)$ & $<0.001$ \\
\hline Statins & $2048(17.1)$ & $590(10.2)$ & $<0.001$ \\
\hline Bisphosphonates & $358(3.0)$ & $199(3.5)$ & 0.108 \\
\hline SSRI & 2009 (16.8) & $723(12.5)$ & $<0.001$ \\
\hline
\end{tabular}

Notes: ${ }^{a}$ Data available for 16,251 patients. ${ }^{b}$ During the two years preceding baseline.

Abbreviations: SD, standard deviation; SEK, Swedish crowns; ICS, inhaled corticosteroids; LAMA, long-acting muscarinic antagonists; LABA, long-acting beta2-agonists; $A C E i$, angiotensin-converting enzyme inhibitors; $A R B$, angiotensin-II receptor blockers; SSRI, selective serotonin reuptake inhibitors. 
burden. Drug usage at baseline did not differ much between the two groups, except for diuretics that were substantially more common among those who died, and statins, SSRI and fixed combination ICS/LABA that were less common among those who died.

\section{Socioeconomic Factors}

In the stepwise multiple Cox model, male gender and higher age were associated with an increased mortality risk, whereas higher income and higher education were associated with a decreased risk; marital status did not influence risk of death (Table 2).

\section{Exacerbations and Comorbidities}

Exacerbations and a high score (at baseline) on the Charlson comorbidity index were associated with an increased risk of death in the stepwise multiple Cox model, as were the comorbidities HF, MI, IHD, stroke, diabetes and fractures, while concomitant asthma and hypertension were associated with a decreased risk of death. Baseline pneumonias were not included in the final multiple Cox model, and there was no statistically significant association between osteoporosis or depression and COPD mortality (Table 3).

\section{Drug Usage}

In the stepwise multiple Cox model adjusting for age, gender, socioeconomic status, exacerbations, comorbidities and any use of the drug classes of interest, the relative exposure to six drug classes reached statistical significance (Table 4, Figure 1). ICS, LAMA and NAC were associated with an increased COPD mortality risk in a dose-dependent manner, whereas beta-blockers, ASA and SSRI were associated with a decreased risk.
In a sensitivity analysis, the relative exposure to LAMA was stratified for concomitant use of ICS (yes or no). Use of LAMA was dose-dependently associated with higher risk of mortality both in COPD patients on LAMA in combination with ICS (HR 1.24, 95\% CI: 1.16-1.32, p: <0.001) and in COPD patients on LAMA but no ICS (HR 1.35, 95\% CI: $1.24-1.47, \mathrm{p}:<0.001)$ when compared to patients not using LAMA.

\section{Discussion}

This study demonstrated that several factors were associated with mortality risk in a large, real-world, primary care COPD population. The strongest predictor of death was comorbid HF, which was associated with a nearly doubled mortality risk. Stroke and MI were also associated with an increased risk of death of about $50 \%$ each. In terms of drug usage, the results indicated a decreased risk of death associated with the use of ICS, betablockers, ASA and SSRI, and an increased risk associated with the use of LAMA and NAC. This study confirms that the negative prognostic role of above-mentioned comorbidities found in previous studies is valid also in a NorthEuropean primary care COPD population. Moreover, the study adds to the previously limited real-world evidence on the impact of drug-usage on COPD mortality.

The association between ICS and decreased mortality seen in this material was in contrast to RCTs and metaanalyses reporting no difference between ICS and placebo with regard to COPD mortality, ${ }^{8,9}$ but was in line with analyses of pooled data ${ }^{25,26}$ and earlier observational data. ${ }^{27}$ In the TORCH study, ${ }^{8}$ there was a statistically nonsignificant tendency towards decreased mortality in the ICS-treated groups. Jenkins et al found in a post-hoc analysis of the TORCH study that treatment with ICS in

Table 2 Demographic/Socioeconomic Factors and Mortality Risk in COPD Patients During the Study Period. Complete Data Available for 16,25I Patients. Analyzed by Simple and Stepwise Multiple (Adjusted for Exacerbations, Comorbidities and Drug Usage) Cox Proportional Hazards Regression Models, Respectively. Empty Fields Indicate Variables Not Included in the Final Stepwise Multiple Cox Model

\begin{tabular}{|l|l|l|l|l|l|l|}
\hline \multirow{2}{*}{} & \multicolumn{2}{l|}{ Simple Cox Regression } & \multicolumn{3}{l|}{ Stepwise Multiple Cox Regression } \\
\cline { 2 - 7 } & HR & $\mathbf{9 5 \%} \mathbf{~ C l}$ & p-value & HR & $\mathbf{9 5 \%}$ Cl & p-value \\
\hline Male gender & 1.30 & $(1.23-1.38)$ & $<0.001$ & 1.16 & $(1.09-1.23)$ & $<0.001$ \\
Age (for every 10 years) & 1.09 & $(1.09-1.09)$ & $<0.001$ & 1.06 & $(1.05-1.06)$ & $<0.001$ \\
Marital status: single & 1.48 & $(1.39-1.58)$ & $<0.001$ & & & \\
Yearly income (per 100,000 SEK) & 0.74 & $(0.70-0.78)$ & $<0.001$ & 0.96 & $(0.92-1.00)$ & 0.040 \\
Education (high vs low) & 0.38 & $(0.35-0.4 I)$ & $<0.001$ & 0.85 & $(0.78-0.92)$ & $<0.001$ \\
\hline
\end{tabular}

Abbreviations: $\mathrm{HR}$, hazard ratio; $\mathrm{Cl}$, confidence interval; SEK, Swedish crowns. 
Table 3 Exacerbations, Pneumonias, Comorbidities and Risk of Mortality in COPD Patients During the Study Period. Complete Data Available for 16,25I Patients. Analyzed by Simple and Stepwise Multiple (Adjusted for Demographic/Socioeconomic Factors and Drug Usage) Cox Proportional Hazards Regression Models, Respectively. Empty Fields Indicate Variables Not Included in the Final Stepwise Multiple Cox Model

\begin{tabular}{|c|c|c|c|c|c|c|}
\hline & \multicolumn{3}{|c|}{ Simple Cox Regression } & \multicolumn{3}{|c|}{ Stepwise Multiple Cox Regression } \\
\hline & HR & $95 \% \mathrm{Cl}$ & p-value & HR & $95 \% \mathrm{Cl}$ & p-value \\
\hline Exacerbations $^{\mathrm{a}}$ & 1.04 & $(1.04-1.04)$ & $<0.001$ & 1.02 & $(1.01-1.02)$ & $<0.001$ \\
\hline Pneumonias ${ }^{\mathrm{b}}$ & 1.44 & $(1.32-1.58)$ & $<0.001$ & & & \\
\hline Heart failure & 4.23 & $(4.00-4.48)$ & $<0.001$ & 1.88 & $(1.74-2.04)$ & $<0.001$ \\
\hline Myocardial infarction & 2.87 & $(2.67-3.07)$ & $<0.001$ & 1.40 & $(1.24-1.58)$ & $<0.001$ \\
\hline Ischemic heart disease & 2.53 & $(2.38-2.69)$ & $<0.001$ & 1.18 & $(1.06-1.32)$ & 0.004 \\
\hline Stroke & 2.56 & $(2.38-2.72)$ & $<0.001$ & 1.52 & $(1.40-1.64)$ & $<0.001$ \\
\hline Hypertension & 1.07 & $(1.01-1.14)$ & 0.021 & 0.90 & $(0.84-0.96)$ & 0.003 \\
\hline Diabetes & 1.37 & $(1.27-1.47)$ & $<0.001$ & 1.13 & $(1.03-1.23)$ & 0.008 \\
\hline Osteoporosis & 1.71 & $(1.56-1.88)$ & $<0.001$ & 1.10 & $(0.97-1.24)$ & 0.137 \\
\hline Fractures $^{\mathrm{a}}$ & 1.17 & $(1.14-1.19)$ & $<0.001$ & 1.07 & $(1.04-1.10)$ & $<0.001$ \\
\hline Depression & 1.00 & $(0.93-1.08)$ & 0.978 & & & \\
\hline Asthma ${ }^{\mathrm{b}}$ & 0.91 & $(0.85-0.98)$ & 0.014 & 0.70 & $(0.64-0.76)$ & $<0.001$ \\
\hline Charlson comorbidity index ${ }^{\mathrm{b}}$ & 1.31 & $(1.29-1.34)$ & $<0.001$ & 1.12 & $(1.09-1.14)$ & $<0.001$ \\
\hline
\end{tabular}

Notes: ${ }^{\text {a Per event. }}{ }^{\mathrm{b}}$ At baseline only.

Abbreviations: $\mathrm{HR}$, hazard ratio; $\mathrm{Cl}$, confidence interval.

combination with a LABA reduced mortality compared to placebo among patients with moderate COPD ${ }^{28}$ Recently, Oshagbemi and coworkers reported retrospective observational real-world data indicating that ICS reduced mortality in COPD patients with blood eosinophilia. ${ }^{29}$ These findings suggest that there might be COPD phenotypes in which ICS can reduce mortality. Heterogeneity of the COPD populations in this and other studies, and the existence of different, today insufficiently understood COPD phenotypes may explain the various results in different studies. Finally, there is also the possibility that in the realworld primary care COPD population, as opposed to the highly select populations in RCTs, ICS might have a positive effect on COPD mortality.

In the present study, we found a dose-dependent relationship between the use of dry powder tiotropium bromide, which was the only LAMA approved in Sweden at the time of the study, and increased mortality. This contrasts with the results from the UPLIFT ${ }^{30} \mathrm{RCT}$ indicating a reduced risk of death. Other studies, including Cochrane reviews, have found no effect at all of LAMA on mortality. ${ }^{11,31,32}$ The increased mortality associated with LAMA treatment reported in the present study may be the result of confounding factors not sufficiently accounted for, for instance lung function or smoking, or bias occurring as a result of the imperfection of the real-world setting, such as diagnoses not properly verified. It is possible that in our material, the group receiving LAMA alone represent the "purest" COPD population and thereby have a higher mortality than the mixed COPD group receiving other kinds of medication. However, an association between the use of LAMA and mortality was also found in the group of patients that were using LAMA in combination with ICS. Our study comprises all real-world COPD patients in a huge primary care population and as such includes a lot of vulnerable and fragile patients seldom included in clinical trials. The effect of LAMA on COPD mortality in this group remains to be further investigated.

$\mathrm{N}$-acetylcysteine may decrease exacerbation rate in $\mathrm{COPD},{ }^{33}$ but has to our knowledge not previously been associated with an increased risk of COPD mortality. There is a possibility that anti-oxidative effects of NAC may play a part. A more plausible explanation, however, for the increased mortality implied here is confounding by chronic bronchitis, which the current study has neither assessed nor adjusted for. Ever since the concept of COPD became widely accepted in Sweden, chronic bronchitis is seldom registered separately but rather included in the COPD-diagnosis. Therefore, we deemed that analyses on chronic bronchitis, based on the present register and medical records data, would be unreliable. Chronic bronchitis is common in COPD, with estimates varying between $14 \%$ and $74 \%$; it is a common reason for prescribing NAC, and it is also a risk factor of death. ${ }^{34}$ Therefore, there are reasons 
Table 4 Drug Usage and Risk of Mortality in COPD Patients During the Study Period, Assessed Both as Any Use of and as Relative Exposure to the Drug Class. Complete Data Available for 16,25I Patients. Analyzed by Simple and Stepwise Multiple (Adjusted for Demographic/Socioeconomic Factors, Exacerbations and Comorbidities) Cox Proportional Hazards Regression Models, Respectively. Empty Fields Indicate Variables Not Included in the Final Stepwise Multiple Cox Model

\begin{tabular}{|c|c|c|c|c|c|c|}
\hline & \multicolumn{3}{|c|}{ Simple Cox Regression } & \multicolumn{3}{|c|}{ Stepwise Multiple Cox Regression } \\
\hline & HR & $95 \% \mathrm{Cl}$ & p-value & HR & $95 \% \mathrm{Cl}$ & p-value \\
\hline \multicolumn{7}{|l|}{ Any Use of Drug Class } \\
\hline ICS & 0.99 & $(0.93-1.05)$ & 0.710 & & & \\
\hline LAMA & 1.43 & $(1.35-1.52)$ & $<0.001$ & 1.07 & $(0.98-1.18)$ & 0.138 \\
\hline LABA & 1.12 & $(1.05-1.19)$ & $<0.001$ & 0.87 & $(0.75-1.00)$ & 0.055 \\
\hline ICS/LABA, fixed combination & 1.18 & $(1.12-1.25)$ & $<0.001$ & & & \\
\hline $\mathrm{N}$-acetylcysteine & 1.18 & $(1.11-1.25)$ & $<0.001$ & 0.90 & $(0.82-0.98)$ & 0.022 \\
\hline Oral steroids & 1.36 & $(1.28-1.44)$ & $<0.001$ & 1.16 & $(1.08-1.25)$ & $<0.001$ \\
\hline Acetylsalicylic acid & 1.77 & $(1.67-1.88)$ & $<0.001$ & 1.29 & $(1.17-1.43)$ & $<0.001$ \\
\hline ACEi & 1.46 & $(1.37-1.56)$ & $<0.001$ & 0.82 & $(0.73-0.93)$ & 0.002 \\
\hline Beta-blockers & 1.37 & $(1.29-1.45)$ & $<0.001$ & & & \\
\hline ARB & 0.94 & $(0.86-1.01)$ & 0.104 & 0.85 & $(0.79-0.92)$ & $<0.001$ \\
\hline Diuretics & 2.78 & $(2.62-2.96)$ & $<0.001$ & 1.36 & $(1.26-1.48)$ & $<0.001$ \\
\hline Statins & 0.75 & $(0.69-0.80)$ & $<0.001$ & 0.65 & $(0.60-0.7 I)$ & $<0.001$ \\
\hline Bisphosphonates & 1.36 & $(1.28-1.44)$ & $<0.001$ & 1.21 & $(1.05-1.40)$ & 0.010 \\
\hline SSRI & 1.34 & $(1.26-1.43)$ & $<0.001$ & 0.82 & $(0.75-0.90)$ & $<0.001$ \\
\hline \multicolumn{7}{|l|}{ Relative Exposure to Drug Class } \\
\hline ICS & 1.10 & $(1.00-1.21)$ & 0.043 & 0.79 & $(0.66-0.94)$ & 0.008 \\
\hline LAMA & 1.73 & $(1.57-1.90)$ & $<0.001$ & 1.33 & $(1.14-1.55)$ & $<0.001$ \\
\hline LABA & 1.16 & $(1.05-1.29)$ & 0.003 & 1.25 & $(0.97-1.6 I)$ & 0.087 \\
\hline ICS/LABA, fixed combination & 1.25 & $(I . \mid I-I .4 I)$ & $<0.001$ & 0.81 & $(0.61-1.08)$ & 0.153 \\
\hline $\mathrm{N}$-acetylcysteine & 1.92 & $(1.72-2.14)$ & $<0.001$ & 1.26 & $(1.08-1.48)$ & 0.004 \\
\hline Oral steroids & 2.81 & $(2.44-3.24)$ & $<0.001$ & & & \\
\hline Acetylsalicylic acid & 2.11 & $(1.92-2.31)$ & $<0.001$ & 0.87 & $(0.77-0.98)$ & 0.022 \\
\hline ACEi & 2.01 & $(1.79-2.25)$ & $<0.001$ & & & \\
\hline Beta-blockers & $\mathrm{I} .44$ & $(1.3|-| .58)$ & $<0.001$ & 0.86 & $(0.76-0.97)$ & 0.016 \\
\hline ARB & 0.90 & $(0.78-1.04)$ & 0.154 & & & \\
\hline Diuretics & 3.61 & $(3.33-3.91)$ & $<0.001$ & & & \\
\hline Statins & 0.69 & $(0.60-0.78)$ & $<0.001$ & & & \\
\hline Bisphosphonates & 1.90 & $(1.5 I-2.39)$ & $<0.001$ & & & \\
\hline SSRI & 1.30 & $(1.13-1.50)$ & $<0.001$ & 0.70 & $(0.56-0.87)$ & 0.001 \\
\hline
\end{tabular}

Abbreviations: HR, hazard ratio; $\mathrm{Cl}$, confidence interval; ICS, inhaled corticosteroids; LAMA, long-acting muscarinic antagonists; LABA, long-acting beta-2-agonists; ACEi, angiotensin-converting enzyme inhibitors; ARB, angiotensin-II receptor blockers; SSRI, selective serotonin reuptake inhibitors.

to believe that NAC, as a marker of chronic bronchitis only, is associated with increased mortality.

Beta-blockers were associated with decreased mortality in the current study, independently of hypertension and heart diseases. Our findings were in line with the majority of previous observational studies reporting a beneficial relationship between beta-blockers and decreased COPD mortality. ${ }^{12,35}$ The present results are, however, in contrast to a recently published RCT where no difference in time to first exacerbation was found between COPD patients treated with metoprolol and COPD patients not treated with metoprolol. ${ }^{36}$ Other RCTs examining the effect of betablockers in COPD are ongoing and positive result from these trials would have the potential to bring a valuable yet inexpensive tool to the COPD treatment arsenal. ${ }^{37}$ The need for such RCTs is highlighted by the fact that fears of prescribing beta-blockers to COPD patients still exist. ${ }^{35}$

Studies indicate that ASA might play a protective role in COPD, regardless of CVD ${ }^{14,38}$ though no RCTs assessing the issue are currently available. The present study supported the idea of a beneficial dose-dependent effect of ASA on COPD mortality. Other possible explanations for the 


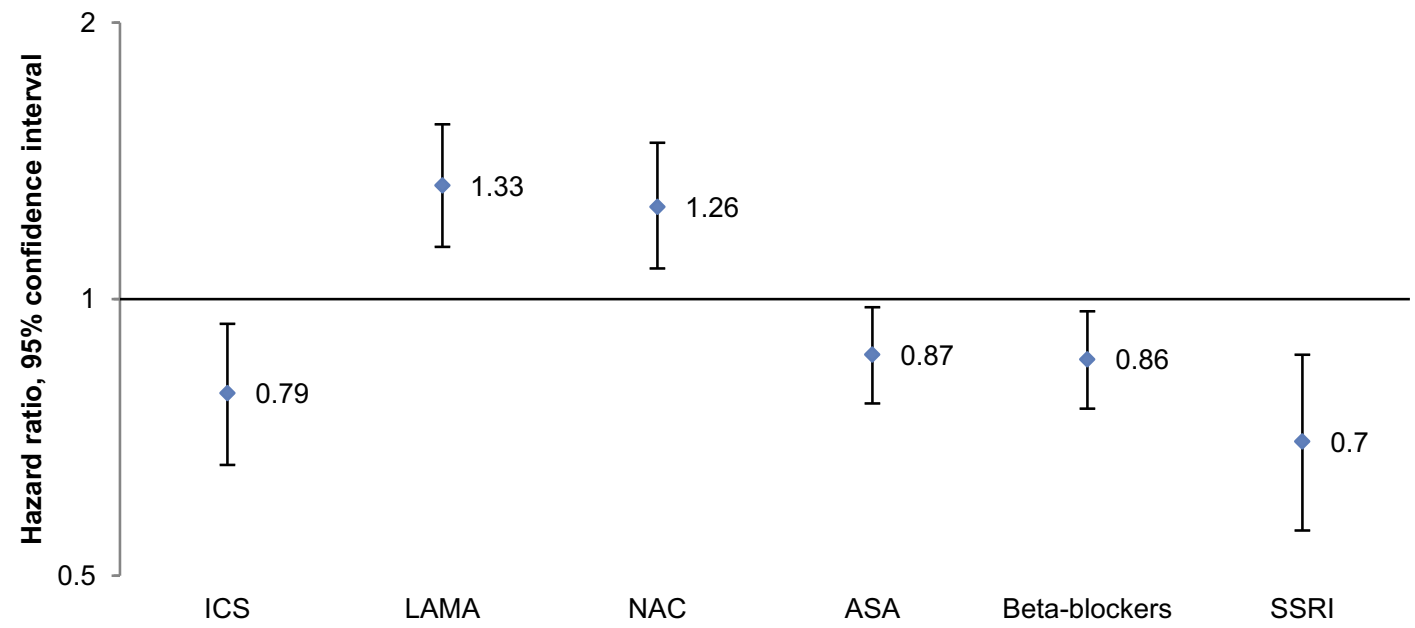

Figure I Stepwise multiple (adjusted for demographic/socioeconomic factors, exacerbations, comorbidities and drug usage - any use) Cox proportional hazards regression model of the effect of drug usage, per relative exposure, on risk of mortality in COPD patients during the study period. Complete data available for I6,25I patients. Abbreviations: ICS, inhaled corticosteroids; LAMA, long-acting muscarinic antagonists; NAC, N-acetylcysteine; ASA, acetylsalicylic acid; SSRI, selective serotonin reuptake inhibitors.

observed association might be under-diagnosis of CVD or incomplete registration in patient records, precluding adjustment for such comorbidities. However, a direct effect of ASA on pathologically activated platelets in COPD patients have been suggested as a potential pathophysiological mechanism. ${ }^{14}$ Recently, 10-year results from the longitudinal MESA Lung Study reported decreased deterioration of pulmonary emphysema in COPD patients treated with ASA, ${ }^{39}$ further supporting the idea of a direct beneficial effect of ASA in COPD.

In our material, SSRI was dose-dependently associated with decreased mortality in COPD. Previous reports have suggested that SSRI might improve depressed COPD patients' quality of life and exercise capacity, ${ }^{40}$ possibly by improving patients' perception of their health status. Our results were contradictory to a recent, large, observational study reporting increased morbidity and mortality with new SSRI use. ${ }^{41}$ However, that study reports no dosedependency, suggesting there might be confounding factors not accounted for. Under-diagnosis of depression is common in $\mathrm{COPD},{ }^{3}$ and may explain our results assuming there were unrecognized and untreated cases of depression, ${ }^{42}$ with increased mortality risk, ${ }^{4}$ in the non-SSRI group.

Several of the negative prognostic factors identified in our study, including age, male gender, exacerbations and comorbidities such as heart disease, stroke, diabetes, fractures and the Charlson comorbidity index score, are known from previous work. ${ }^{4-7,43-45}$ The positive prognostic factors reported here (higher education and income) are also in line with previous studies. ${ }^{46}$ Our results confirm the prognostic value of the above-mentioned factors also in the real-world primary care setting.

The present study found, after adjustment for use of antihypertensive drugs, an association between hypertension and a decreased risk of mortality, which contrasts with previous works reporting no association with mortality. ${ }^{5,16}$ There is, to our knowledge, no pathophysiological mechanism that could explain a protective role of hypertension in terms of COPD mortality. Potential explanations to our results include that hypertension might have been underdiagnosed, leading to relatively more deaths among unrecognized and untreated subjects in the non-hypertension group, and beneficial effects of antihypertensive drugs not sufficiently adjusted for (ie, calcium channel blockers).

A diagnosis of asthma before COPD diagnosis substantially decreased the risk of mortality in the present analysis. While some investigators have found increased mortality in COPD patients with concurrent asthma, ${ }^{47}$ most studies have reported a mortality rate lower than ${ }^{48}$ or similar to ${ }^{49}$ subjects with COPD only. These conflicting results might possibly be explained by differences between the studies in how and when diagnoses of asthma and COPD have been assessed and verified. They may also represent the clinical reality of the two diagnoses not always being readily defined and separated.

There were several strengths of this study; the main ones being its size, long follow-up time and coverage representing about $8 \%$ of the Swedish population. By combining data 
from patient records and mandatory national registers, this study encompassed virtually all patients with a COPD diagnosis visiting any of the included primary care centers during the study period. In the selection of the centers included, the aim was to get a sample representative of Swedish primary care in terms of geographic and demographic properties as well as private and public care providers. Therefore, the study population is likely to be representative of Swedish primary care COPD patients, and the results are likely to be generalizable. The use of national registers based on the personal identification number guaranteed complete data of good quality, insensitive of subjects moving around. Another strength of this study was the plethora of data collected, enabling analysis of many possible risk factors and adjustment for a wide range of variables.

The most important limitation, except for the intrinsic limitations of the observational study setting, is the lack of objective confirmation of most of the diseases included in the analysis. In other words, all diseases may be both under-diagnosed (or incompletely registered in patient records) and over-diagnosed. The lack of data on smoking history was another major limitation, since smoking is a major risk factor not only for COPD but also for CVD and early death, among others. Data on smoking history could also have strengthened the diagnosis of COPD, and to some extent helped in the distinction between COPD and asthma. Another major limitation of this study was the lack of data on lung function, limiting the possibilities to verify the COPD-diagnosis and to stratify data per lung function. If the relative risk of mortality increased with increasing COPD severity ("dose-dependency"), the association with possible risk factors would have been strengthened. Yet another limitation was the lack of data on blood eosinophil numbers, which have been reported to be prognostic of COPD mortality. Information on the vaccination status of the subjects would have been useful, since vaccination for respiratory infections has a wellestablished protective effect in COPD and therefore likely would influence mortality estimates. Data on body mass index, a well-established risk factor for COPD death as well as for CVD, were also lacking in this study.

\section{Conclusion}

This observational study of a large cohort of real-world primary care COPD patients indicated a decreased risk of death associated with the use of ICS, beta-blockers and
ASA, and an increased risk associated with the use of LAMA and NAC. Chronic bronchitis might explain the increased mortality risk associated with NAC. The results highlight the need for further studies, preferably RCTs, on the effect and safety of ICS, beta-blockers and ASA on different COPD phenotypes. The strongest predictors of death were comorbid heart failure, stroke and myocardial infarction. Timely recognition and appropriate treatment of these and other comorbidities may improve prognosis, underscoring the importance of a multidimensional and thorough assessment of COPD patients in primary as well as secondary care.

\section{Abbreviations}

ACEi, angiotensin-converting enzyme inhibitor; ARB, angiotensin-II receptor blocker; ASA, acetylsalicylic acid; ATC, Anatomical Therapeutic Chemical classification system; CI, confidence interval; COPD, chronic obstructive pulmonary disease; CVD, cardiovascular disease; HF, heart failure; HR, hazard ratio; ICD-10, International Classification of Diseases, 10th edition; ICS, inhaled corticosteroids; IHD, ischemic heart disease; MI, acute myocardial infarction; LABA, long-acting beta-2-agonist; LAMA, long-acting muscarinic antagonist; NAC, N-acetylcysteine; RCT, randomized controlled trial; SD, standard deviation; SEK, Swedish crowns; SSRI, selective serotonin reuptake inhibitor.

\section{Ethics Approval}

The regional ethics committee in Uppsala, Sweden, reviewed and approved the study protocol (reference number 2010/040) and did not require patient consent since all data were anonymized.

\section{Data Sharing Statement}

Data will continue to be held and managed by the Department of Public Health and Caring Sciences, Uppsala University, Uppsala, Sweden. According to the European Union's General Data Protection Regulation, data from this study are classified as sensitive and must not be made available through an open repository. Anonymized patient-level data will be made available from the corresponding author after ethical approval.

\section{Acknowledgment}

This manuscript was in part made possible thanks to grants from Uppsala County Association against Heart and Lung Diseases. 


\section{Author Contributions}

All authors made substantial contributions to conception and design, acquisition of data, or analysis and interpretation of data; took part in drafting the article or revising it critically for important intellectual content; gave final approval of the version to be published; and agree to be accountable for all aspects of the work. JE drafted and finalized the manuscript. MT was responsible for handling of data and the study database and for the statistical analyses. $\mathrm{CJ}$ is guarantor.

\section{Funding}

This study was financially supported by AstraZeneca.

\section{Disclosure}

JE has received honoraria for lectures from AstraZeneca, Novartis and Teva and has served on advisory boards arranged by Novartis. GJ has served on advisory boards arranged by AstraZeneca, Novartis and Teva. KjL has, during the last five years, on one or more occasion served as an advisory board member and/or served as speaker and/or participated in education activities arranged by AstraZeneca, Boehringer Ingelheim, Chiesi, GlaxoSmithKline, Orion, Novartis, Teva and Takeda. KaL has received honoraria for educational activities from Novartis, AstraZeneca, Chiesi, Teva and Boehringer Ingelheim and served on advisory boards arranged by Novartis and GlaxoSmithKline. AM has no conflicts of interest to declare with regard to the topic of the present study. BS has received honoraria for educational activities and lectures from AstraZeneca, Boehringer Ingelheim, GlaxoSmithKline, Novartis, Meda and Teva, and has served on advisory boards arranged by AstraZeneca, Novartis, GlaxoSmithKline, Boehringer Ingelheim, Meda and Teva. MT has received personal fees from AstraZeneca, during the conduct of the study. CJ has received honoraria for educational activities from AstraZeneca, Boehringer Ingelheim, Chiesi, Novartis and Teva, and has served on advisory boards arranged by AstraZeneca, Boehringer Ingelheim, Chiesi, GlaxoSmithKline, Novartis and Teva. The authors report no other conflicts of interest in this work.

\section{References}

1. Naghavi M, Abajobir AA, Abbafati C, et al. Global, regional, and national age-sex specific mortality for 264 causes of death, 1980-2016: a systematic analysis for the Global Burden of Disease Study 2016. Lancet. 2017;390(10100):1151-1210. doi:10.1016/S01406736(17)32152-9
2. Stallberg B, Janson C, Johansson G, et al. Management, morbidity and mortality of COPD during an 11-year period: an observational retrospective epidemiological register study in Sweden (PATHOS). Prim Care Respir J. 2014;23(1):38-45. doi:10.4104/pcrj.2013.00106

3. Global Initiative for Chronic Obstructive Lung Disease (GOLD). From the global strategy for the diagnosis, management and prevention of chronic obstructive lung disease, 2019 report. Available from: http://goldcopd.org. Accessed September 2, 2019.

4. Divo M, Cote C, de Torres JP, et al. Comorbidities and risk of mortality in patients with chronic obstructive pulmonary disease. Am J Respir Crit Care Med. 2012;186:155- 161.

5. Miller J, Edwards LD, Agusti A, et al. Comorbidity, systemic inflammation and outcomes in the ECLIPSE cohort. Respir Med. 2013;107 (9):1376-1384. doi:10.1016/j.rmed.2013.05.001

6. Baty F, Putora PM, Isenring B, Blum T, Brutsche M. Comorbidities and burden of COPD: a population based case-control study. PLoS One. 2013;8(5):e63285. doi:10.1371/journal.pone.0063285

7. Stallberg B, Janson C, Larsson K, et al. Real-world retrospective cohort study ARCTIC shows burden of comorbidities in Swedish COPD versus non-COPD patients. NPJ Prim Care Respir Med. 2018;28(1):33. doi:10.1038/s41533-018-0101-y

8. Calverley PM, Anderson JA, Celli B, et al. Salmeterol and fluticasone propionate and survival in chronic obstructive pulmonary disease. $N$ Engl J Med. 2007;356(8):775-789. doi:10.1056/NEJMoa063070

9. Vestbo J, Anderson JA, Brook RD, et al. Fluticasone furoate and vilanterol and survival in chronic obstructive pulmonary disease with heightened cardiovascular risk (SUMMIT): a double-blind randomised controlled trial. Lancet. 2016;387(10030):1817-1826. doi:10.1016/s0140-6736(16)30069-1

10. Kew KM, Mavergames C, Walters JA. Long-acting beta2-agonists for chronic obstructive pulmonary disease. Cochrane Database Syst Rev. 2013;10:Cd010177. doi:10.1002/14651858.CD010177.pub2

11. Karner C, Chong J, Poole P. Tiotropium versus placebo for chronic obstructive pulmonary disease. Cochrane Database Syst Rev. 2014;7: CD009285. doi:10.1002/14651858.CD009285.pub3

12. Du Q, Sun Y, Ding N, Lu L, Chen Y. Beta-blockers reduced the risk of mortality and exacerbation in patients with COPD: a meta-analysis of observational studies. PLoS One. 2014;9:e113048. doi:10.1371/ journal.pone. 0113048

13. Mortensen EM, Copeland LA, Pugh MJ, et al. Impact of statins and ACE inhibitors on mortality after COPD exacerbations. Respir Res. 2009;10:45. doi:10.1186/1465-9921-10-45

14. Pavasini R, Biscaglia S, d'Ascenzo F, et al. Antiplatelet treatment reduces all-cause mortality in COPD patients: a systematic review and meta-analysis. Copd. 2016;13(4):509-514. doi:10.3109/ 15412555.2015.1099620

15. Criner GJ, Connett JE, Aaron SD, et al. Simvastatin for the prevention of exacerbations in moderate-to-severe COPD. $N$ Engl J Med. 2014;370(23):2201-2210. doi:10.1056/NEJMoa1403086

16. Miniati M, Monti S, Pavlickova I, Bottai M. Survival in COPD: impact of lung dysfunction and comorbidities. Medicine (Baltimore). 2014;93(12):e76. doi:10.1097/MD.0000000000000076

17. Sidney S, Sorel M, Quesenberry CP Jr, DeLuise C, Lanes S, Eisner MD. COPD and incident cardiovascular disease hospitalizations and mortality: Kaiser Permanente Medical Care Program. Chest. 2005;128(4):2068-2075. doi:10.1378/chest.128.4.2068

18. Larsson K, Janson C, Lisspers K, et al. Combination of budesonide/ formoterol more effective than fluticasone/salmeterol in preventing exacerbations in chronic obstructive pulmonary disease: the PATHOS study. J Intern Med. 2013;273(6):584-594. doi:10.1111/joim.2013.273. issue- 6

19. Janson C, Larsson K, Lisspers KH, et al. Pneumonia and pneumonia related mortality in patients with COPD treated with fixed combinations of inhaled corticosteroid and long acting beta2 agonist: observational matched cohort study (PATHOS). BMJ. 2013;346:f3306. doi:10.1136/bmj.f1164 
20. Lisspers $\mathrm{K}$, Johansson $\mathrm{G}$, Jansson $\mathrm{C}$, et al. Improvement in COPD management by access to asthma/COPD clinics in primary care: data from the observational PATHOS study. Respir Med. 2014;108 (9):1345-1354. doi:10.1016/j.rmed.2014.06.002

21. Sandelin M, Mindus S, Thuresson M, et al. Factors associated with lung cancer in COPD patients. Int J Chron Obstruct Pulmon Dis. 2018;13:1833-1839. doi:10.2147/COPD.S162484

22. Martinell M, Stålhammar J, Hallqvist J. Automated data extraction a feasible way to construct patient registers of primary care utilization. Ups J Med Sci. 2012;117(1):52-56. doi:10.3109/03009734.2011.653015

23. Charlson ME, Pompei P, Ales KL, MacKenzie CR. A new method of classifying prognostic comorbidity in longitudinal studies: development and validation. $J$ Chronic Dis. 1987;40(5):373-383. doi:10.1016/0021-9681(87)90171-8

24. Quan H, Sundararajan V, Halfon P, et al. Coding algorithms for defining comorbidities in ICD-9-CM and ICD-10 administrative data. Med Care. 2005;43(11):1130-1139. doi:10.1097/01. mlr.0000182534.19832.83

25. Sin DD, Wu L, Anderson JA, et al. Inhaled corticosteroids and mortality in chronic obstructive pulmonary disease. Thorax. 2005;60(12):992-997. doi:10.1136/thx.2005.045385

26. Halpin DMG, Peterson S, Larsson TP, Calverley PMA. Identifying COPD patients at increased risk of mortality: predictive value of clinical study baseline data. Respir Med. 2007;102(11):1615-1624. doi:10.1016/j.rmed.2008.05.007

27. Macie C, Wooldrage K, Manfreda J, Anthonisen NR. Inhaled corticosteroids and mortality in COPD. Chest. 2006;130(3):640-646. doi:10.1378/chest.130.3.640

28. Jenkins CR, Jones PW, Calverley PM, et al. Efficacy of salmeterol/ fluticasone propionate by GOLD stage of chronic obstructive pulmonary disease: analysis from the randomised, placebo-controlled TORCH study. Respir Res. 2009;10(1):59. doi:10.1186/1465-9921-10-59

29. Oshagbemi OA, Franssen FME, Braeken DCW, et al. Blood eosinophilia, use of inhaled corticosteroids, and risk of COPD exacerbations and mortality. Pharmacoepidemiol Drug Saf. 2018;27 (11):1191-1199. doi:10.1002/pds.4655

30. Celli B, Decramer M, Kesten S, et al. Mortality in the 4-year trial of tiotropium (UPLIFT) in patients with chronic obstructive pulmonary disease. Am J Respir Crit Care Med. 2009;180(10):948-955. doi:10.1164/rccm.200906-0876OC

31. Halpin DM, Dahl R, Hallmann C, Mueller A, Tashkin D. Tiotropium HandiHaler((R)) and $\operatorname{Respimat}((\mathrm{R}))$ in COPD: a pooled safety analysis. Int J Chron Obstruct Pulmon Dis. 2015;10:239-259. doi:10.2147/COPD.S75146

32. Wise RA, Anzueto A, Cotton D, et al. Tiotropium respimat inhaler and the risk of death in COPD. $N$ Engl $J$ Med. 2013;369 (16):1491-1501. doi:10.1056/NEJMoa1303342

33. Poole P, Sathananthan K, Fortescue R. Mucolytic agents versus placebo for chronic bronchitis or chronic obstructive pulmonary disease. Cochrane Database Syst Rev. 2019;5. doi:10.1002/ 14651858

34. Lindberg A, Sawalha S, Hedman L, Larsson LG, Lundback B, Ronmark E. Subjects with COPD and productive cough have an increased risk for exacerbations and death. Respir Med. 2015;109 (1):88-95. doi:10.1016/j.rmed.2014.12.001
35. Andell P, Erlinge D, Smith JG, et al. $\beta$-blocker use and mortality in COPD patients after myocardial infarction: a Swedish nationwide observational study. $J$ Am Heart Assoc. 2015;4(4):e001611. doi:10.1161/JAHA.114.001611

36. Dransfield MT, Voelker H, Bhatt SP, et al. Metoprolol for the prevention of acute exacerbations of COPD. $N$ Engl J Med. 2019;381:2304-2314. doi:10.1056/NEJMoa1908142

37. Heddini A, Sundh J, Ekström M, Janson C. Effectiveness trials: critical data to help understand how respiratory medicines really work? Eur Clin Respir J. 2019;6(1):1565804. doi:10.1080/ 20018525.2019.1565804

38. Ekstrom MP, Hermansson AB, Strom KE. Effects of cardiovascular drugs on mortality in severe chronic obstructive pulmonary disease. Am J Respir Crit Care Med. 2013;187(7):715-720. doi:10.1164/ rccm.201208-1565OC

39. Aaron CP, Schwartz JE, Hoffman EA, et al. A longitudinal cohort study of aspirin use and progression of emphysema-like lung characteristics on CT imaging: the MESA lung study. Chest. 2018;154 (1):41-50. doi:10.1016/j.chest.2017.11.031

40. He Y, Zheng Y, Xu C, et al. Sertraline hydrochloride treatment for patients with stable chronic obstructive pulmonary disease complicated with depression: a randomized controlled trial. Clin Respir J. 2016;10(3):318-325. doi:10.1111/crj.12219

41. Vozoris NT, Wang X, Austin PC, et al. Serotonergic antidepressant use and morbidity and mortality among older adults with COPD. Eur Respir J. 2018;52:1. doi:10.1183/13993003.00475-2018

42. Mignogna J, Cully J. Depression and anxiety in patients with COPD: a focus on psychological treatments in ambulatory care settings. Curr Respir Med Rev. 2012;8:137-144. doi:10.2174/157339812799859743

43. Yamauchi Y, Yasunaga H, Sakamoto Y, et al. Mortality associated with bone fractures in COPD patients. Int J Chron Obstruct Pulmon Dis. 2016;11:2335-2340. doi:10.2147/COPD.S112142

44. Anthonisen NR, Wright EC, Hodgkin JE. Prognosis in chronic obstructive pulmonary disease. Am Rev Respir Dis. 1986;133 (1):14-20. doi:10.1164/arrd.1986.133.1.14

45. de Torres JP, Cote CG, Lopez MV, et al. Sex differences in mortality in patients with COPD. Eur Respir J. 2009;33(3):528-535. doi:10.1183/09031936.00096108

46. Gershon AS, Dolmage TE, Stephenson A, Jackson B. Chronic obstructive pulmonary disease and socioeconomic status: a systematic review. Copd. 2012;9(3):216-226. doi:10.3109/ 15412555.2011.648030

47. Lange P, Çolak Y, Ingebrigtsen TS, Vestbo J, Marott JL. Long-term prognosis of asthma, chronic obstructive pulmonary disease, and asthma-chronic obstructive pulmonary disease overlap in the Copenhagen City Heart study: a prospective population-based analysis. Lancet Respir Med. 2016;4(6):454-462. doi:10.1016/ S2213-2600(16)00098-9

48. Cosio BG, Soriano JB, López-Campos JL, et al. Defining the asthma-COPD overlap syndrome in a COPD cohort. CHEST. 2016;149(1):45-52. doi:10.1378/chest.15-1055

49. Sorino C, Pedone C, Scichilone N. Fifteen-year mortality of patients with asthma-COPD overlap syndrome. Eur $J$ Intern Med. 2016;34:72-77. doi:10.1016/j.ejim.2016.06.020

International Journal of Chronic Obstructive Pulmonary Disease

Dovepress

\section{Publish your work in this journal}

The International Journal of COPD is an international, peer-reviewed journal of therapeutics and pharmacology focusing on concise rapid reporting of clinical studies and reviews in COPD. Special focus is given to the pathophysiological processes underlying the disease, intervention programs, patient focused education, and self management protocols. This journal is indexed on PubMed Central, MedLine and CAS. The manuscript management system is completely online and includes a very quick and fair peer-review system, which is all easy to use. Visit http://www.dovepress.com/testimonials.php to read real quotes from published authors. 